\title{
Future perspective of CAS in orthopaedics
}

\author{
S. Zaffagnini ${ }^{1,2}$ (1) $\cdot$ K. Deep ${ }^{3} \cdot$ N. Confalonieri ${ }^{4}$
}

Received: 2 June 2016 / Accepted: 5 July 2016 / Published online: 12 July 2016

(C) European Society of Sports Traumatology, Knee Surgery, Arthroscopy (ESSKA) 2016

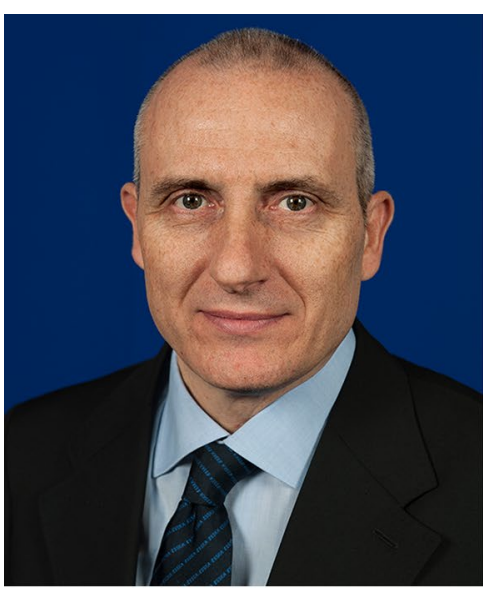

S. Zaffagnini

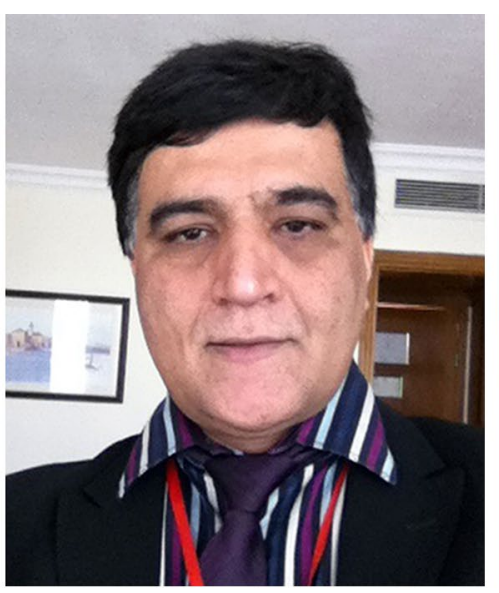

K. Deep

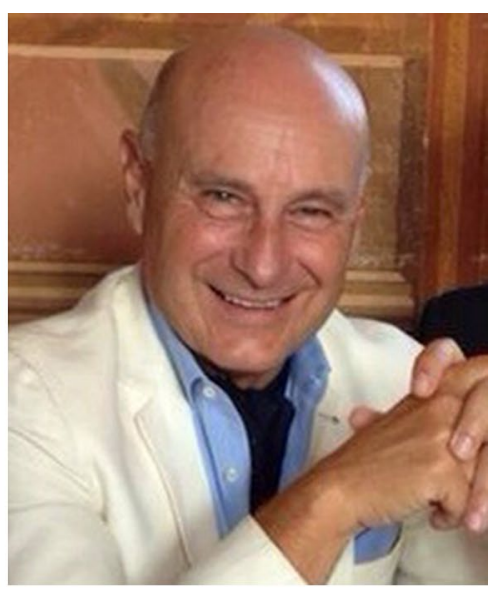

N. Confalonieri
Computer-assisted surgery (CAS) in orthopaedics was officially introduced in 1991, at the Muller's laboratories (Bern), when Professor Nolte inserted the first pedicle screw in a saw bone using a computer navigator [10]. Then, it spread widely especially in France and Germany; in 1997, in Grenoble centre, Picard and Saragaglia introduced the first image-free system for total knee replacement [11].

\section{S. Zaffagnini}

stefano.zaffagnini@unibo.it

1 Laboratorio di Biomeccanica e Innovazione Tecnologica, Istituto Ortopedico Rizzoli, Bologna, BO, Italy

2 Clinica Ortopedica e Trumatologica I, Istituto Ortopedico Rizzoli, Bologna, BO, Italy

3 Department of Orthopaedics, Golden Jubilee National Hospital, Glasgow, UK

4 Reparto di Ortopedia e Traumatologia, Ospedale C.T.O, Milano, MI, Italy
Nowadays navigation is considered a useful tool for in vivo research, for standardization and for the control of surgical prosthetic implantation especially, in complicated cases. Still, the numbers in which this technique can be used is much less with respect to the advantages that its use can achieve. This is mainly due to expensive cost and user friendliness of the system.

According to an American study, there will be a $60 \%$ increase in the number of revisions of joint replacements between 2005 and 2030, but there will not be enough surgeons in the world to manage this increase. A recent study, from the British joint registry of implants published in the Lancet [8], shows that the unicompartmental knee replacement (UKR) is an excellent operation. It leads to early functional improvement and recovery with fewer complications and mortality, compared to total knee replacement. But this includes a higher revisions rate.

The aim, from now on, is to work towards decreasing the number and severity of revisions, with less and 
less-invasive prosthesis, implanted with a more precise technique. In fact, the three major causes of failures are related to wear, loosening and instability, and this is due to a problem of imbalance and malalignment, both related to surgical technique.

CAS was developed to assist surgeons dealing with the various phases of [6] osteoarthritis deformities, ligaments balance, assessment of pre- and post-operative pathology and the control of surgical acts in the operating room.

Several authors have published research showing CAS effectiveness in total joint replacement for both anatomical accuracy and functional improvement. Recently, an Australian registry study that has also shown that the survival in patients less than 65 years of age has increased. This study, which included all grades of surgeons and all the navigation systems used in Australia, brings a strong evidence on how computer navigation is reducing the number of revisions in younger patients [4]. The problems previously quoted with navigation are the longer surgical time and cost for hospitals and companies [12]. For small implants using computer assistance and robotics, there are few studies, mainly in unicompartmental knee replacement, showing similar results $[2,6,9]$.

Today, the world of CAS is varied, starting with the planning of the surgery that requires specific radiological images software running on the computer. The cutting guides, patient specific jigs require a computerised CT or MRI study for their realisation [13].

The robot, after the aggressive steps of the first models, is moving towards less bulky solutions, with more precise software, although the costs remain high. This has increased the number of surgeons, especially the younger one, attracted towards this technology [1, 5].

The indications and scope of applications have been expanded. Navigation in ACL and osteotomies around the knee offer important cues and considerations [7]. Finally, the revision of the prosthesis using computer assistance allows control of the joint line, even when the bone is missing. It gives an excellent aid for the correct implantation [3]. The use of computers is slowly expanding also into the oncological orthopaedic surgery where it can save the limbs and joints. Further improvements in the development of less expensive and less-invasive computer systems as well as the use of new technologies like inertial sensors will provoke a further adoption of CAS, improving clinical outcome and documentation of our results.

The conclusion that can be agreed is that CAS is a technology that improves the clinical results thus improving the precision and accuracy of surgery; therefore, it should be considered part of the usual surgery instrumentation not as a plus.

\section{References}

1. Banerjee S, Cherian JJ, Elmallah RK, Jauregui JJ, Pierce TP, Mont MA (2015) Robotic-assisted knee arthroplasty. Expert Rev Med Devices 12:727-735

2. Confalonieri N, Manzotti A, Montironi F, Pullen C (2008) Tissue sparing surgery in knee reconstruction: unicompartmental (UKA), patellofemoral (PFA), UKA + PFA, biunicompartmental (Bi-UKA) arthroplasties. J Orthop Traumatol 9:171-177

3. Confalonieri N, Manzotti A, Chemello C, Cerveri P (2010) Computer-assisted revision of failed unicompartmental knee arthroplasty. Orthopedics 33:52-57

4. de Steiger RN, Liu Y-L, Graves SE (2015) Computer navigation for total knee arthroplasty reduces revision rate for patients less than 65 years of age. J Bone Joint Surg Am 97:635-642

5. Goradia VK (2014) Computer-assisted and robotic surgery in orthopedics: where we are in 2014. Sports Med Arthrosc Rev 22:202-205

6. Jenny J-Y (2008) The current status of computer-assisted high tibial osteotomy, unicompartmental knee replacement, and revision total knee replacement. Instr Course Lect 57:721-726

7. Koh J, Marcus MS (2014) Computer-assisted anterior cruciate ligament (ACL) reconstruction: the US perspective. Sports Med Arthrosc Rev 22:206-214

8. Liddle AD, Judge A, Pandit H, Murray DW (2014) Adverse outcomes after total and unicompartmental knee replacement in 101,330 matched patients: a study of data from the National Joint Registry for England and Wales. Lancet Lond Engl 384:1437-1445

9. Manzotti A, Cerveri P, Pullen C, Confalonieri N (2014) Computer-assisted unicompartmental knee arthroplasty using dedicated software versus a conventional technique. Int Orthop 38:457-463

10. Nolte LP, Zamorano LJ, Jiang Z, Wang Q, Langlotz F, Berlemann U (1995) Image-guided insertion of transpedicular screws. A laboratory set-up. Spine 20:497-500

11. Saragaglia D, Picard F, Chaussard C, Montbarbon E, Leitner F, Cinquin P (2001) Computerassisted knee arthroplasty: comparison with a conventional procedure. Results of 50 cases in a prospective randomized study. Rev Chir Orthop Réparatrice Appar Mot 87:18-28

12. Swank ML, Alkire M, Conditt M, Lonner JH (2009) Technology and cost-effectiveness in knee arthroplasty: computer navigation and robotics. Am J Orthop (Belle Mead NJ) 38:32-36

13. Thienpont E, Schwab PE, Fennema P (2014) A systematic review and meta-analysis of patient specific instrumentation for improving alignment of the components in total knee replacement. Bone Joint J 96-B:1052-1061 